\title{
CORRIGENDUM
}

\section{Direct to consumer genetic tests}

\author{
Christine Patch, Jorge Sequeiros and Martina C Cornel
}

European Journal of Human Genetics (2009) 17, 1204; doi:10.1038/ejhg.2009.110

Correction to: European Journal of Human Genetics (2009) 17, 1111; doi:10.1038/ejhg.2009.66; published online 29 April 2009

Since the publication of the above paper, the authors have noticed that the authors' affiliations are wrong.

The correct affiliations are listed below.

Christine Patch ${ }^{1,2}$, Jorge Sequeiros ${ }^{1,3}$ and Martina C Cornel ${ }^{1,4}$

${ }^{1}$ Public and Professional Policy Committee of the European Society of Human Genetics, Vienna, Austria; ${ }^{2}$ Department of Clinical Genetics, NIHR Biomedical Research Centre, Guys and St Thomas' NHS Foundation Trust, Guys Hospital, London, UK; ${ }^{3}$ IBMC - Instituto de Biologia Molecular e Celular, and ICBAS, University of Porto, Porto, Portugal; ${ }^{4}$ Community Genetics, Department of Clinical Genetics/EMGO Institute, VU University Medical Center, Amsterdam, The Netherlands

The authors would like to apologise for this mistake. 